\title{
Terahertz wave generation from a dc-biased multimode laser
}

\author{
Sylwester Latkowski, Frederic Surre, ${ }^{\text {a) }}$ and Pascal Landais \\ School of Electronic Engineering, Dublin City University, Glasnevin, Dublin 9, Ireland
}

(Received 6 September 2007; accepted 25 January 2008; published online 28 February 2008)

\begin{abstract}
We present results achieved in the generation of terahertz wave by a semiconductor laser. It is a Fabry-Pérot based device with shallow grooves implemented on its $p$-side to engineer the longitudinal mode spectrum. The laser is dc-biased and temperature controlled at $298 \mathrm{~K}$. The main two modes are separated by $3 \mathrm{~nm}$ at $1550 \mathrm{~nm}$ with a side-mode-suppression ratio of $25 \mathrm{~dB}$. Using a frequency resolved optical gating, evidence of mode beating at $373 \mathrm{GHz}$ is observed. With a bolometer interfaced to a Fourier transform interferometer, the second harmonic signal is measured at $690 \mathrm{GHz}$. (C) 2008 American Institute of Physics. [DOI: 10.1063/1.2884525]
\end{abstract}

Terahertz spectrum is confined between $300 \mathrm{GHz}$ and $10 \mathrm{THz}$, i.e., between the radio-frequency and the infrared domains. Although there are many applications for terahertz radiations (e.g., biology and biological imaging, manufacturing, quality control, and security/antiterrorist screening), schemes to generate these waves are quite limited. One of these techniques is free electron laser, which provides the highest power but at a very high cost. ${ }^{1}$ An alternative is to use Schottky resonant tunnel diode oscillator array. This is a complex structure generating a fixed relative low oscillation frequency. ${ }^{2} P$-type germanium lasers ${ }^{3}$ and quantum cascade lasers ${ }^{4}$ require cryogenic cooling temperatures in order to produce terahertz emission. The photoconductive switch exploits the photocurrent generated from ultrashort pulses in a semiconductor antenna. ${ }^{5}$ This method is commonly used between 0.5 and $5 \mathrm{THz}$. Another approach is to use a photonic transmitter. Two dc laser optical signals ${ }^{6}$ (or one pulse signal filtered ${ }^{7}$ ) are launched into a photonic transmitter coupled to an antenna. The signals beat together in the photomixer and the intermediate frequency is emitted by the antenna. This technique runs at room temperature and, by changing the frequency of the signals, the beating signal can be tuned. In this paper, we propose a solution in which the terahertz signal is generated by a multimode semiconductor laser at room temperature. At least two optical signals beat together inside the laser cavity generating a signal at the intermediate frequency and, in our case, in the terahertz regime. In our approach, the terahertz signal generated benefit from a good overlap between the spatial modes and also from the nonlinear effects in the semiconductor material that is able to respond to a terahertz excitation. Compared to the photonic transmitter, our solution does not require any external source. Therefore, the solution is more stable and its footprint is smaller.

Intracavity mode beating in dc biased multimode FabryPérot (FP) semiconductor lasers has been demonstrated as an easy way to generate fluctuations of the output signal at a frequency of $100 \mathrm{GHz}$ corresponding to the mode detuning. ${ }^{8}$ The optical field generated inside the laser cavity can be expressed as a monochromatic wave with a slowly time varying amplitude

\footnotetext{
${ }^{a)}$ Electronic mail: surref@eeng.dcu.ie.
}

$$
E_{k}(z, t)=A_{k}(t) \exp \left\{-j\left[\omega_{k} t+\phi k(t)\right]\right\} Z_{k}(z),
$$

where $A_{k}$ is the amplitude of the $k$ th mode, $\omega_{k}$ its angular frequency, $Z_{k}$ its longitudinal dependence, and $\varphi_{k}$ its instantaneous phase fluctuation. In the case of a laser with $M$ longitudinal modes, the beating between these modes leads to a quadratic temporal average of the total electric field with the following expression:

$$
\begin{aligned}
\left\langle\left|E_{T}\right|^{2}\right\rangle= & \sum_{k=1}^{M}\left\langle\left|E_{k}\right|^{2}\right\rangle+\sum_{k=1}^{M} \sum_{j \neq k} 2\left\langleE _ { k } E _ { j } \operatorname { c o s } \left\{\Omega_{k j} t+\left[\varphi_{j}(t)\right.\right.\right. \\
& \left.\left.\left.-\varphi_{k}(t)\right]\right\}\right\rangle,
\end{aligned}
$$

where the beating frequency between two adjacent modes $\Omega_{k j}$ is given by $\left(\omega_{j}-\omega_{k}\right)$. For example, for a laser with two uncorrelated modes, a beating signal at the frequency $\Omega_{k j} / 2 \pi$ can be observed by a photodiode, with a spectral linewidth corresponding to the sum of the two modes' spectral linewidths. The double sum in Eq. (2) is equal to zero, as $\varphi_{k}$ and $\varphi_{j}$ vary independently from each other. Quite recently, it has been demonstrated that for multimode distributed Bragg reflector lasers with a free-spectral range of $40 \mathrm{GHz}$, the rf signal generated through mode beating due to carrier density pulsation (CDP) has a smaller linewidth than that of the optical longitudinal modes beating. It was proven that the phase of rf signal benefits from passive phase mode locking resulting from CDP. ${ }^{9}$ In this case, the double sum in Eq. (2) is different from zero. CDP is the main nonlinearity inherent in semiconductor lasers that can produce mode beating and, under favorable conditions, phase correlation at $40 \mathrm{GHz}$. It is generally accepted that CDP is the dominant nonlinearity until about $100 \mathrm{GHz}$. Between $100 \mathrm{GHz}$ and $1 \mathrm{THz}$, carrier heating $(\mathrm{CH})$ becomes dominant. Above $1 \mathrm{THz}$, both $\mathrm{CDP}$ and $\mathrm{CH}$ are weak and only spectral hole burning (SHB) can produce mode beating. In theory, it seems possible to generate a periodic modulation of the optical output of a multimode laser at frequency beyond $300 \mathrm{GHz}$ by exploiting mode beating due to material nonlinearities such as $\mathrm{CH}$ and $\mathrm{SHB}$.

The approach to assert this hypothesis is to use a multimode laser where dominant mode wavelengths are separated by a few nanometers within the $3 \mathrm{~dB}$ gain spectrum. This will lead to a beating frequency of some hundreds of terahertz. One interesting solution from an application point of view, due to its cheap cost of production, is a FP laser. If the length of the cavity is appropriately selected, it should be 


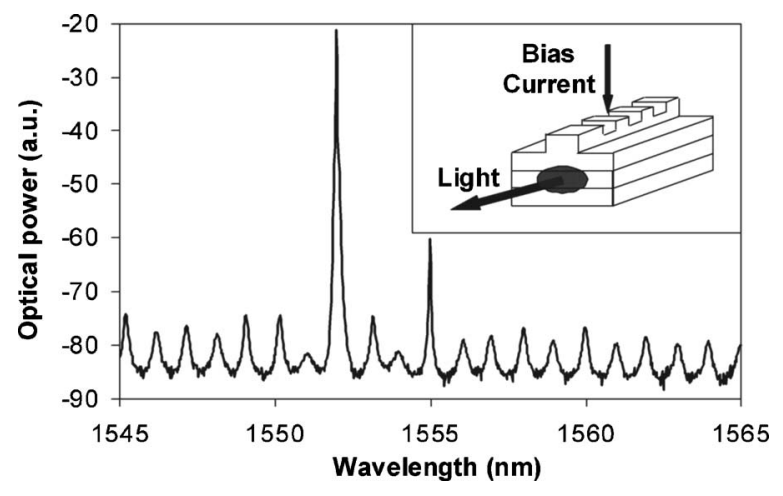

FIG. 1. Optical spectrum of the slotted laser for $I=60 \mathrm{~mA}$ and $T=298 \mathrm{~K}$. Inset: schematic of slotted laser structure.

possible to achieve a free-spectral range from some hundreds of gigahertz to a few terahertz. Assuming a refractive index of 3.5 for the active layer, the length of the device will be $50 \mu \mathrm{m}$ and with associated mirror losses of $200 \mathrm{~cm}^{-1}$. The threshold current density over the active layer is in the order of $1.5 \times 10^{9} \mathrm{~A} / \mathrm{cm}^{2} \mu \mathrm{m}$, well above the usual value of $5 \mathrm{kA} / \mathrm{cm}^{2} \mu \mathrm{m}$. Another approach, also based on a FP laser, is to implement a one-dimensional photonic band gap in the laser cavity. The easiest way is to etch shallow grooves on the $p$-side of the laser junction perpendicular to the longitudinal axis of the device, ${ }^{10}$ as depicted in the inset of Fig. 1. These grooves are not deep enough to reach the active layer, but they are deep enough to affect the effective refractive index experienced by the electromagnetic wave generated in the active layer. By etching these grooves at key positions, it is possible to generate variations of the refractive index along the cavity and, consequently, to control the longitudinal spectrum. The FP resonance determines an initial spectral separation between consecutive modes, but the etched band gap suppresses or enhances some specific FP modes. It is feasible, therefore, to select modes to achieve a given freespectral range. In this case, we are aiming for a detuning in the terahertz range corresponding to a Free Spectral Range (FSR) between 2.5 and $10 \mathrm{~nm}$ at $1550 \mathrm{~nm}$.

The advantages of this type of device are many. The production cost is low, the footprint is small, and power consumption moderate. The shallow grooves are not etched through the active layer. Hence, the device is not multisection and does not require several bias currents. The fact that the mode beating takes place within the laser cavity, and not from an extended external cavity ${ }^{11}$ or from the mixing of two laser sources, ${ }^{6}$ results in a more mechanically and thermally stable source apparatus. In our experiments, the laser is temperature controlled at $298 \mathrm{~K}$ and dc biased. It is a $350 \mu \mathrm{m}$ long multiquantum well InAlGaAs FP laser. Its $2 \mu \mathrm{m}$ ridge waveguide provides a spatial single mode output. The grooves are $1 \mu \mathrm{m}$ deep and $2 \mu \mathrm{m}$ wide. The prototype device is characterized by a current threshold of $I_{\mathrm{th}}=19 \mathrm{~mA}$. Figure 1 shows an optical spectrum at a bias current of $60 \mathrm{~mA}$. The resolution of the optical spectrum analyzer is $0.05 \mathrm{~nm}$. The ripples are separated by $1 \mathrm{~nm}$ corresponding to a free-spectral range of about $120 \mathrm{GHz}$ at $1550 \mathrm{~nm}$ and are due to the spurious FP modes of the $350 \mu \mathrm{m}$ cavity. The groove pattern designed on this device enhances a main mode at $1551.98 \mathrm{~nm}$ and a second mode at $1554.98 \mathrm{~nm}$ resulting with a side-mode-suppression ratio of approximately $39 \mathrm{~dB}$ in these experimental conditions of bias current and Downloaded 28 Apr 2008 to 136.206.1.17. Redistribution subject to

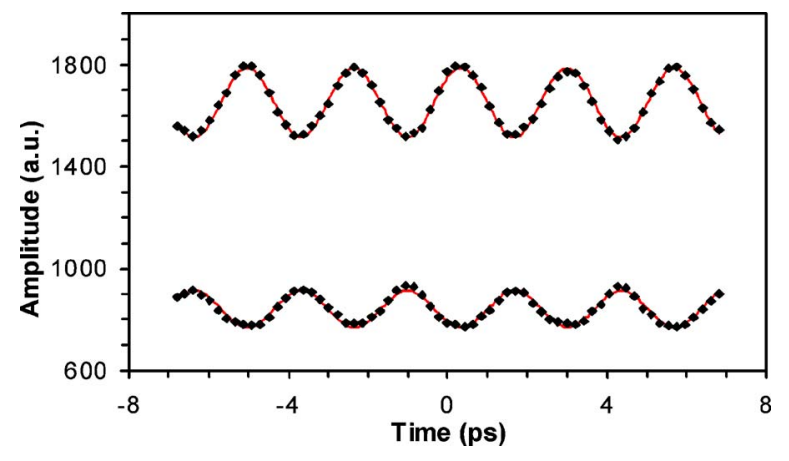

FIG. 2. (Color online) (Density of data points reduced for clarity.) Time variation of the amplitude of the first two modes as a function of time measured with a FROG. The laser is biased at $89.73 \mathrm{~mA}$ and its temperature is set at $298 \mathrm{~K}$. The crosses correspond to the experimental measurements and the continuous line to the fit of sinusoidal wave. The origin of time is arbitrary.

temperature. As the laser spectrum exhibits two modes, it is theoretically possible to have a mode beating between them, leading to the creation of a terahertz signal with the frequency $373 \mathrm{GHz}$. Furthermore, the optical power associated with each mode will fluctuate with the same frequency as the beating signal.

In order to observe the beating and, therefore, evidence of terahertz signal, the time variation of both modes has been recorded using a frequency resolved optical gating (FROG) over a time period of $15 \mathrm{ps}$, as presented in Fig. 2. The temporal resolution is $107 \mathrm{fs}$ and the wavelength resolution is $24 \mathrm{pm}$. The laser is biased at $I=89.73 \mathrm{~mA}$ and temperature controlled at $298 \mathrm{~K}$. Two main modes are detected. The experimental data are represented by black crosses and are fitted using a sinusoidal function with a nonzero mean, drawn by a red full line. Two parameters of interest can be extracted from this interpolation. The phase difference between modes and the frequency of the modulation of each signal. The phase difference calculated is $\sim \pi$ radians. If the modulation was applied to the laser diode or to the laser output, both signals will be in phase. In this case, the signals are in opposition phase. This demonstrates that the modulation is originated by an intracavity mode beating. The extracted modulation frequencies are $373.24 \mathrm{GHz}$ for the main mode and $373.14 \mathrm{GHz}$ for the second mode. The modulation frequency gives a direct estimation of the beating frequency. Both values of frequency are in good agreement with the experimental value of the FSR estimated from the optical spectrum.

Under the same experimental conditions as that of Fig. 1 , the laser emission has been analyzed using a Fourier transform (FT-IR) spectrometer equipped with a bolometer. The laser beam waves are evenly split into two beams traveling along independent paths inside the spectrometer. The length of one of the paths with respect with the other is varied using a moveable mirror. The interference pattern is produced by recombining both beams. Finally, the interferometric signal is collimated to a bolometer cooled at $3.6 \mathrm{~K}$ for detection. The energy detected by the bolometer is recorded as a function of the path difference. After zero filling, the interferogram recorded is fast Fourier transformed using an apodization function in order to suppress the side lobes created by the Fast Fourier Transform. The result of this processing is the spectrum of the injected signal. The beam splitters used allowed signals from $400 \mathrm{GHz}$ to $1 \mathrm{THz}$ to be transmitted in AIP license or copyright; see http://apl.aip.org/apl/copyright.jsp 

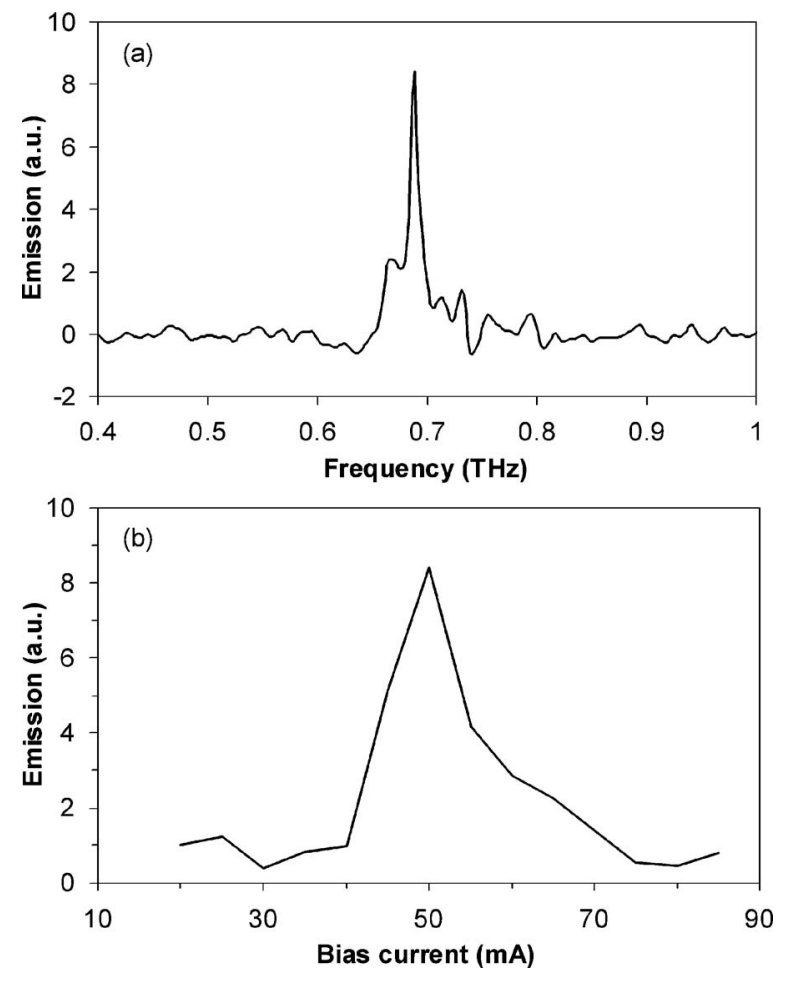

FIG. 3. (a) Terahertz spectrum for a bias current of $50 \mathrm{~mA}$. (b) Evolution of the terahertz signal for the second harmonic at $690 \mathrm{GHz}$ as a function of the bias current. For both figures the device is temperature controlled at $298 \mathrm{~K}$.

order to filter out the IR emission from the laser. Hence, it is expected that only the terahertz signal is launched into the detector. There is no possible detection of a $370 \mathrm{GHz}$ wave; however, any signal above $400 \mathrm{GHz}$ can be analyzed. All spectra presented are recorded with a resolution of $0.06 \mathrm{~cm}^{-1}$ which corresponds to a resolution of $1.8 \mathrm{GHz}$. For each bias current measurement, we apply the same modus operandi. The signal, detected by the bolometer with the laser switched off, is recorded and fast Fourier transformed. This spectrum is stored under label $I_{0}$. Then, the laser is switched on. A new interferogram is recorded at the bolometer. The spectrum associated with this interferogram is stored under label $I$. The emission is defined by the ratio $\left(I-I_{0}\right) / I_{0}$. Figure 3 (a) presents the terahertz spectrum for a bias current of $50 \mathrm{~mA}$ and a temperature of $298 \mathrm{~K}$ corresponding to the maximum terahertz signal detected. The frequency of the terahertz peak is at $700 \mathrm{GHz}$ approximately, which corresponds to the second harmonic of the beating signal, taking into account measurement and calibration errors. The peak is approximately ten times larger than the level of noise detected by this setup. As the beating is enhanced by the phase correlation between optical modes, the linewidth of the terahertz signal, either first or second harmonic, is at most equal to the sum of the linewidth of both optical modes. These linewidths have been measured using a heterodyne technique ${ }^{12}$ at $30 \mathrm{MHz}$. Due to the spectrometer resolution of $1.8 \mathrm{GHz}$ and the small linewidth of the terahertz signal, it is not possible to find the exact value of the terahertz signal. The value presented in Fig. 3(a) is a rough estimation of this maximum. The actual peak is located between two sampled frequencies and is ex- pected to be significantly higher than ten times the noise level.

Figure 3(b) presents the evolution of the second harmonic signal as a function of the bias current of the laser. The current varies from 20 to $90 \mathrm{~mA}$. Below $40 \mathrm{~mA}$, it is not possible to detect a signal strong enough to emerge from the noise level. As the bias current increases, the terahertz signal gets stronger and reaches a peak at $50 \mathrm{~mA}$ [cf. Fig. 3(a)]. One has to keep in mind that, due to the resolution of the FT-IR spectrometer, the peak value is only an approximation. After $50 \mathrm{~mA}$, it appears that the detected signal decreases with bias current. No conclusion should be taken out of this result. One must remember that the resolution of the apparatus is $1.8 \mathrm{GHz}$ and the spectral width of the terahertz signal is at most equal to the sum of the optical linewidths. The linewidth of the terahertz signal is expected to decrease with bias current. As the resolution of the experiment is still $1.8 \mathrm{GHz}$, it is increasingly difficult to have a point close to the maximum and so the estimated value of the terahertz signal is greatly undervalued. This can explain the decrease observed on Fig. 3(b). Furthermore, the redshift of the first harmonic, estimated from the variation of the FSR from the optical spectrum, is below $1 \mathrm{GHz}$ and the redshift of the second harmonic is below the resolution of the experiment.

In this paper, we demonstrated the generation of a terahertz signal exploiting the mode beating in a multimode laser temperature controlled at $298 \mathrm{~K}$. The device is based on a FP laser with grooves implemented along the active layer to control the longitudinal spectrum. It has been possible, from the recorded variation of the optical spectrum with a FROG, to estimate the frequency of the beating signal. Furthermore, the measurement of the phase difference between the two main modes showed that the optical power modulation was created by mode beating inside the laser cavity. Finally, the use of FT-IR spectrometer has led to the measurement of a signal at $690 \mathrm{GHz}$ with a contrast ratio of approximately 10 . This signal is the second harmonic of the FSR set by the groove pattern at $370 \mathrm{GHz}$.

This project is supported by SFI Research Frontier programme referenced 05/RFP/ENG0040. The authors would like to thank Dr. Stephen A. Lynch for his help with the FT-IR experiment.

${ }^{1}$ G. L. Carr, M. C. Martin, W. R. McKinney, K. Jordan, G. R. Neil, and G. P. Williams, Nature (London) 420, 153 (2002).

${ }^{2}$ M. Reddy, S. C. Martin, A. C. Molnar, R. E. Muller, R. P. Smith, P. H. Siegel, M. J. Mondry, M. J. W. Rodwell, H. Kroemer, and S. J. Allen, Jr., IEEE Electron Device Lett. 18, 218 (1997).

${ }^{3}$ E. Brundermann, A. M. Linhart, H. P. Roser, O. D. Dubon, W. L. Hansen, and E. E. Haller, Appl. Phys. Lett. 68, 1359 (1996).

${ }^{4}$ C. Shun Lien, IEEE J. Quantum Electron. 38, 510 (2002).

${ }^{5}$ D. H. Auston, K. P. Cheung, and P. R. Smith, Appl. Phys. Lett. 45, 284 (1984).

${ }^{6}$ S. M. Duffy, S. Verghese, A. McIntosh, A. Jackson, A. C. Gossard, and S. Matsuura, IEEE Trans. Microwave Theory Tech. 49, 1032 (2001).

${ }^{7}$ A. S. Weling, B. B. Hu, N. M. Froberg, and D. H. Auston, Appl. Phys. Lett. 64, 137 (1994).

${ }^{8}$ K. Sato, Electron. Lett. 37, 763 (2001).

${ }^{9}$ J. Renaudier, G. H. Duan, P. Landais, and P. Gallion, IEEE J. Quantum Electron. 43, 147 (2007).

${ }^{10}$ P. Landais, Patent No. S2005/0251 (Ireland, 26 April 2005).

${ }^{11}$ S. Hoffmann, X. Luo, and M. Hofmann, Electron. Lett. 42, 696 (2006).

${ }^{12}$ T. Okoshi, K. Kikuchi, and A. Nakayama, Electron. Lett. 16, 630 (1980). 